\title{
Parabosons, parafermions, and explicit representations of infinite-dimensional algebras
}

\author{
N.I. Stoilova ${ }^{1}$ and J. Van der Jeugt ${ }^{1}$ \\ ${ }^{1}$ Department of Applied Mathematics and Computer Science, Ghent University, Belgium
}

The goal of this paper is to give an explicit construction of the Fock spaces of the parafermion and the paraboson algebra, for an infinite set of generators. This is equivalent to constructing certain unitary irreducible lowest weight representations of the (infinite rank) Lie algebra $\mathfrak{s o}(\infty)$ and of the Lie superalgebra $\mathfrak{o s p}(1 \mid \infty)$. A complete solution to the problem is presented, in which the Fock spaces have basis vectors labeled by certain infinite but stable Gelfand-Zetlin patterns, and the transformation of the basis is given explicitly. Alternatively, the basis vectors can be expressed as semi-standard Young tableaux.

PACS numbers: $03.65 .-\mathrm{w}, 03.65 . F d, 02.20 .-\mathrm{a}, 11.10 .-\mathrm{z}$

\section{INTRODUCTION}

Parafermions and parabosons [1] were introduced in order to study particle statistics of a more general type than the common Fermi-Dirac and Bose-Einstein statistics. These generalizations have an algebraic formulation in terms of generators and relations $[1,2]$. The parafermion operators $f_{j}^{ \pm}, j=1,2, \ldots$ (generalizing the statistics of spinor fields), satisfy certain triple commutation relations, see (1), and it has been known for a long time [3, 4] that the Lie algebra generated by these operators subject to the defining triple relations is an orthogonal Lie algebra. More precisely, for a finite set of parafermions $(j=1,2, \ldots, n)$ it is the Lie algebra $\mathfrak{s o}(2 n+1)$. In the context of quantum field theory, however, one is mainly interested in the case of an infinite set of parafermions, $j \in \mathbb{Z}_{+}=\{1,2, \ldots\}$. The corresponding parafermion algebra is an infinite rank Lie algebra, often referred to as $\mathfrak{s o}(\infty)$.

The paraboson operators $b_{j}^{ \pm}, j=1,2, \ldots$ (generalizing the statistics of tensor fields), also satisfy certain triple relations, see (4), this time however involving anticommutators and commutators. It was therefore logical to look for a connection with Lie superalgebras instead of with Lie algebras. This connection was established in [5], and the Lie superalgebra 
generated by a finite set $(j=1,2, \ldots, n)$ of paraboson operators subject to the defining triple relations is the orthosymplectic Lie superalgebra $\mathfrak{o s p}(1 \mid 2 n)$. For an infinite set of parabosons, the paraboson algebra is an infinite rank Lie superalgebra denoted as $\mathfrak{o} \mathfrak{s p}(1 \mid \infty)$.

The main objects of interest are the unitary lowest weight representations of the relevant algebras with a nondegenerate lowest weight space (i.e. with a unique vacuum). These representations are precisely the so-called Fock spaces of the parafermion or paraboson algebras. These Fock spaces are characterized by a positive integer $p$, often referred to as the order of statistics. The Fock spaces $W(p)$ (for parafermions) and $V(p)$ (for parabosons) can be defined in many ways, and one of the standard methods is due to Green and known as Green's ansatz $[1,2]$. Due to computational difficulties, Green's ansatz has actually not lead to an explicit construction of the Fock spaces. An explicit construction of the parafermion or paraboson Fock spaces was not known until recently (by an explicit construction is meant: giving a complete orthogonal basis of the Fock space and the explicit action of the parafermion or paraboson operators on these basis vectors). For the case of a finite set of parafermions, this explicit construction was given in [6], and for a finite set of parabosons in [7].

In the present paper, we extend the results of $[6,7]$ to the case of an infinite set of generators. This is not trivial, as the transition from finite rank algebras to infinite rank algebras gives rise to some complications.

\section{THE PARAFERMION AND PARABOSON ALGEBRAS}

We define a Lie algebra and a Lie superalgebra by means of a set of generators and a set of relations. In both cases, the set of generators is an infinite set.

Definition 1 Let $\mathfrak{g}=\mathfrak{s o}(\infty)$ be the (complex) Lie algebra with generators $\left\{f_{j}^{-}, f_{j}^{+} \mid j \in \mathbb{Z}_{+}=\right.$ $\{1,2, \ldots\}\}$ and relations

$$
\left[\left[f_{j}^{\xi}, f_{k}^{\eta}\right], f_{l}^{\epsilon}\right]=\frac{1}{2}(\epsilon-\eta)^{2} \delta_{k l} f_{j}^{\xi}-\frac{1}{2}(\epsilon-\xi)^{2} \delta_{j l} f_{k}^{\eta}
$$

where $j, k, l \in \mathbb{Z}_{+}$and $\eta, \epsilon, \xi \in\{+,-\}$ (to be interpreted as +1 and -1 in the algebraic expressions $\epsilon-\xi$ and $\epsilon-\eta)$.

The relations (1) are the defining triple relations for a system of parafermions [1], which is why $\mathfrak{g}$ is often called the parafermion algebra. For a finite set of such generators $(j \in$ 
$\{1,2, \ldots, n\})$ and the corresponding relations, this Lie algebra is the orthogonal Lie algebra $\mathfrak{s o}(2 n+1)$, see $[3,4,6]$. This explains the notation $\mathfrak{s o}(\infty)$ for $n=\infty$.

Using the defining relations (1), it is easy to give a basis of $\mathfrak{g}$ :

$$
f_{j}^{ \pm} \quad\left(j \in \mathbb{Z}_{+}\right), \quad\left[f_{j}^{ \pm}, f_{k}^{ \pm}\right] \quad\left(j<k ; j, k \in \mathbb{Z}_{+}\right), \quad\left[f_{j}^{+}, f_{k}^{-}\right] \quad\left(j, k \in \mathbb{Z}_{+}\right) .
$$

So, as a vector space, $\mathfrak{g}$ consist of all finite linear combinations of the elements (2) with complex coefficients. Consider now the elements $h_{j}=\frac{1}{2}\left[f_{j}^{+}, f_{j}^{-}\right]\left(j \in \mathbb{Z}_{+}\right)$and the subspace $\mathfrak{h}$ of $\mathfrak{g}$ spanned by these elements. This is the Cartan subalgebra of $\mathfrak{g}$.

Let $\mathfrak{h}^{*}$ be the vector space dual to $\mathfrak{h}$, with dual basis $\left\{\epsilon_{j} \mid j \in \mathbb{Z}_{+}\right\}$defined by $\epsilon_{j}\left(h_{k}\right)=\delta_{j k}$. All the elements from (2) are root vectors $x_{\alpha}$ of $\mathfrak{g}$, in the sense that $\left[h_{j}, x_{\alpha}\right]=\alpha\left(h_{j}\right) x_{\alpha}$ for all $j \in \mathbb{Z}_{+}$, for some $\alpha \in \mathfrak{h}^{*}$. The roots $\alpha$ corresponding to the elements (2) are given by

$$
\pm \epsilon_{j}\left(j \in \mathbb{Z}_{+}\right), \quad \pm\left(\epsilon_{j}+\epsilon_{k}\right) \quad\left(j<k ; j, k \in \mathbb{Z}_{+}\right), \quad \epsilon_{j}-\epsilon_{k} \quad\left(j, k \in \mathbb{Z}_{+}\right) .
$$

The set of nonzero roots is thus $\Delta=\left\{ \pm \epsilon_{j}, \pm\left(\epsilon_{j}+\epsilon_{k}\right)(j<k), \epsilon_{j}-\epsilon_{k}(j \neq k)\right\}$. So $\mathfrak{g}$ has a root space decomposition, and one can verify (e.g. from the commutation relation between root vectors) that $\mathfrak{g}$ coincides with the Lie algebra $\mathfrak{g}^{\prime}\left(B_{\infty}\right)$ as defined by Kac $[8, \S 7.11]$, sometimes also referred to as "the Lie algebra $B_{\infty}$ ".

For us, it will be useful to have the parafermion creation operators as positive root vectors, and the annihilation operators as negative root vectors. So we define the set of positive roots as $\Delta_{+}=\left\{\epsilon_{j},\left(\epsilon_{j}+\epsilon_{k}\right)(j<k), \epsilon_{j}-\epsilon_{k}(j<k)\right\}$ and $\Delta_{-}$, the opposite set, as the set of negative roots. With $\mathfrak{n}_{+}$(resp. $\mathfrak{n}_{-}$) defined as the subalgebra spanned by the positive (resp. negative) root vectors, $\mathfrak{g}$ has the usual triangular decomposition $\mathfrak{g}=\mathfrak{n}_{-} \oplus \mathfrak{h} \oplus \mathfrak{n}_{+}$. Note, however, that with the current choice of $\Delta_{+}$it is not possible to give a set of simple roots.

Now we shall define a closely related algebra $\mathfrak{g}$, which is however a Lie superalgebra [9] rather than a Lie algebra. So this second algebra has a $\mathbb{Z}_{2}$-grading, $\mathfrak{g}=\mathfrak{g}_{0} \oplus \mathfrak{g}_{\overline{1}}, \mathfrak{g}_{0}$ being the even elements and $\mathfrak{g}_{\overline{1}}$ being the odd elements, with the usual "supercommutation relation" (which is, in particular, an anticommutator $\{\cdot, \cdot\}$ when both elements are odd).

Definition 2 Let $\mathfrak{g}=\mathfrak{o s p}(1 \mid \infty)$ be the (complex) Lie superalgebra with odd generators $\left\{b_{j}^{-}, b_{j}^{+} \mid j \in \mathbb{Z}_{+}\right\}$and relations

$$
\left[\left\{b_{j}^{\xi}, b_{k}^{\eta}\right\}, b_{l}^{\epsilon}\right]=(\epsilon-\xi) \delta_{j l} b_{k}^{\eta}+(\epsilon-\eta) \delta_{k l} b_{j}^{\xi},
$$

where $j, k, l \in \mathbb{Z}_{+}$and $\eta, \epsilon, \xi \in\{+,-\}$ (to be interpreted as +1 and -1 in the algebraic expressions $\epsilon-\xi$ and $\epsilon-\eta)$. 
The relations (4) are the defining triple relations for a system of parabosons [1], and $\mathfrak{g}$ is often called the paraboson algebra. For a finite set of such generators $(j \in\{1,2, \ldots, n\})$ and the corresponding relations, the resulting Lie superalgebra is the orthosymplectic Lie superalgebra osp $(1 \mid 2 n)$, see [5, 7].

Once again, using the defining relations (4), one finds a basis of $\mathfrak{g}$ :

$$
b_{j}^{ \pm} \quad\left(j \in \mathbb{Z}_{+}\right), \quad\left\{b_{j}^{ \pm}, b_{k}^{ \pm}\right\} \quad\left(j<k ; j, k \in \mathbb{Z}_{+}\right), \quad\left\{b_{j}^{+}, b_{k}^{-}\right\} \quad\left(j, k \in \mathbb{Z}_{+}\right) .
$$

In fact, $\mathfrak{g}_{\overline{1}}$ is spanned by the elements $b_{j}^{ \pm}\left(j \in \mathbb{Z}_{+}\right)$, and $\mathfrak{g}_{\overline{0}}$ by the remaining elements of (5). Let us define the (even) elements $h_{j}=\frac{1}{2}\left\{b_{j}^{+}, b_{j}^{-}\right\}\left(j \in \mathbb{Z}_{+}\right)$and the (even) subspace $\mathfrak{h}$ of $\mathfrak{g}$ spanned by these elements. One can again verify that $\mathfrak{h}$ is a Cartan subalgebra of $\mathfrak{g}$. The dual vector space $\mathfrak{h}^{*}$ is defined as before, with dual basis $\left\{\epsilon_{j} \mid j \in \mathbb{Z}_{+}\right\}$. All elements from (5) are root vectors $x_{\alpha}$ of $\mathfrak{g}$, for some $\alpha \in \mathfrak{h}^{*}$. The roots $\alpha$ corresponding to the elements (5) are given by the same set (3). This time, the set of nonzero roots is $\mathbb{Z}_{2}$-graded, and one speaks of the even roots $\Delta_{0}=\left\{ \pm\left(\epsilon_{j}+\epsilon_{k}\right)(j<k), \epsilon_{j}-\epsilon_{k}(j \neq k)\right\}$ and the odd roots $\Delta_{1}=\left\{ \pm \epsilon_{j}\right\}\left(\Delta=\Delta_{0} \cup \Delta_{1}\right)$. The set of positive roots is defined as before, and the odd and even positive roots are, resp. $\Delta_{1,+}=\left\{\epsilon_{j}\right\}, \Delta_{0,+}=\left\{\left(\epsilon_{j}+\epsilon_{k}\right)(j<k), \epsilon_{j}-\epsilon_{k}(j<k)\right\}$, with $\Delta_{+}=\Delta_{1,+} \cup \Delta_{0,+}$. The opposite set $\Delta_{-}$is the set of negative roots, and $\mathfrak{g}$ has a triangular decomposition.

\section{GZ-PATTERNS, PARTITIONS AND YOUNG TABLEAUX}

Let us define infinite GZ-patterns, inspired by Gelfand-Zetlin patterns for $\mathfrak{u}(n)[10]$.

Definition 3 A GZ-pattern $\mid m$ ) is an infinite table or triangular array of nonnegative integers $m_{i j}\left(i, j \in \mathbb{Z}_{+}, i \leq j\right)$, arranged as follows:

$$
\mid m)=\left(\begin{array}{lcll}
\vdots & \vdots & \ldots & \vdots \\
m_{1 n} & m_{2 n} & \cdots & m_{n n} \\
\vdots & \vdots & . \cdot & \\
m_{12} & m_{22} & & \\
m_{11} & & &
\end{array}\right)=\left(\begin{array}{l}
\vdots \\
{[m]^{n}} \\
\vdots \\
{[m]^{2}} \\
{[m]^{1}}
\end{array}\right)
$$

such that the integer entries satisfy the following betweenness conditions

$$
m_{i, j+1} \geq m_{i j} \geq m_{i+1, j+1} \quad(\text { for all } i \leq j)
$$


As already indicated in (6), we will denote the rows of $\mid m)$ as $[m]^{1}=\left[m_{11}\right],[m]^{2}=\left[m_{12}, m_{22}\right]$, $[m]^{3}=\left[m_{13}, m_{23}, m_{33}\right]$, etc., and refer to this as the first row, second row, third row etc. (we always count the rows from bottom to top). So the $n$th row $[m]^{n}$ consists of a sequence of nonincreasing nonnegative integers of length $n$. Sometimes, it will be convenient to view $[m]^{n}$ as an infinite sequence by adding zeros to the finite sequence.

The infinite GZ-patterns that are relevant for us are those that stabilize after a number of rows, counting from the bottom.

Definition 4 A GZ-pattern $\mid m)$ is stable if there exists a row index $N \in \mathbb{Z}_{+}$such that

$$
[m]^{n}=[m]^{N}, \quad \text { for all } n>N
$$

In such a case, one also says that $\mid m$ ) is stable with respect to row $N$.

Note that, in order to speak of equal rows as in (8), one uses the just mentioned extension by zeros. For a stable GZ-pattern, it is not necessary to give an infinite table: giving just the first $N$ rows is sufficient. To indicate that one is still dealing with infinite GZ-patterns, we put an arrow above the entries of row $N$. For example, the following GZ-pattern, which is stable with respect to row 3 , is denoted by:

$$
\left.\left.\mid \begin{array}{l}
:::::: \vdots \\
53000 \\
5300 \\
530 \\
31 \\
2
\end{array}\right)=\mid \begin{array}{l}
\uparrow \uparrow \uparrow \\
530 \\
31 \\
2
\end{array}\right)
$$

Sometimes it will be useful to consider just the pattern consisting of the first $n$ rows of $\mid m$ ) only. We denote this by

$$
(m)^{n}=\left|\begin{array}{lccc}
m_{1 n} & m_{2 n} & \cdots & m_{n n} \\
\vdots & \vdots & . & \\
m_{12} & m_{22} & & \\
m_{11} & & &
\end{array}\right|
$$

and shall refer to it as a $\mathfrak{u}(n)$ GZ-pattern, as it coincides with the patterns in the common GZ-basis of $\mathfrak{u}(n)[10]$.

Let us also collect some notation on partitions and tableaux, following Macdonald [11]. A partition $\lambda$ is a (finite or infinite) sequence $\lambda=\left(\lambda_{1}, \lambda_{2}, \ldots\right)$ of non-negative integers in decreasing order, $\lambda_{1} \geq \lambda_{2} \geq \cdots$, containing only finitely many non-zero terms. The Young 
diagram of a partition $\lambda$ is the set of left-adjusted rows of squares with $\lambda_{i}$ squares (or boxes) in the $i$ th row, reading now from top to bottom. For example, the Young diagram $F^{\lambda}$ of $\lambda=(5,3,1,1)$ is given by

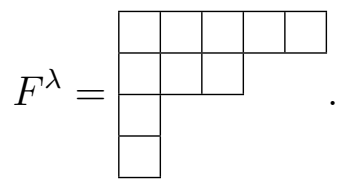

Definition 5 A semi-standard Young tableau (SSYT) of shape $\lambda$, with $\lambda$ a partition, is a numbering of the boxes of the Young diagram of $\lambda$ such that the numbers increase strictly down each column and increase weakly along each row.

For example, for $\lambda=(5,3,1,1)$, the following is a SSYT:

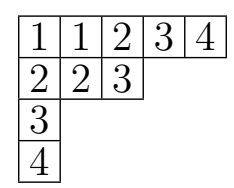

There is a natural bijection between stable GZ-patterns and semi-standard Young tableaux. To see this, note that the rows $[m]^{i}$ of a GZ-pattern can be viewed as a sequence of partitions $[m]^{1} \subseteq[m]^{2} \subseteq \cdots \subseteq[m]^{N}$, such that each skew diagram $[m]^{i} /[m]^{i-1}$ is a horizontal strip [11]; this last condition is due to (7). Then the corresponding semi-standard tableau is obtained by putting the number $i$ in each box of $[m]^{i} /[m]^{i-1}$. For example, for the stable GZ-pattern (9) the sequence of partitions is

$$
(2) \subseteq(3,1) \subseteq(5,3,0),
$$

and so we form a tableau of shape $(5,3,0)$ with 1 's in the part corresponding to the partition (2), with 2's in the part corresponding to the partition $(3,1)$, and with 3's in the part corresponding to the partition $(5,3,0)$, finally leading to:

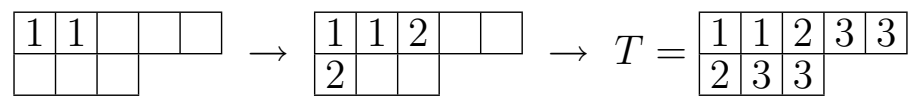

Conversely, each semi-standard Young tableau leads to a stable GZ-pattern by reversing the above process. For example, the tableau (12) yields the following stable GZ-pattern:

$$
\left.\begin{array}{|l}
\uparrow \uparrow \uparrow \uparrow \\
5311 \\
431 \\
32 \\
2
\end{array}\right)
$$

The correspondence between $\mid m$ ) and $T$ can be described in another way: $m_{i j}$ is equal to the number of entries $\leq j$ in row $i$ of the Young tableau $T$. 


\section{PARAFERMION AND PARABOSON FOCK SPACES}

In [2], the parafermion Fock space $W(p)$ of order $p$ is defined, for any positive integer $p$. It is the Hilbert space with unique vacuum vector $|0\rangle$, defined by means of $(j, k=1,2, \ldots)$

$$
\langle 0 \mid 0\rangle=1, \quad f_{j}^{-}|0\rangle=0, \quad\left[f_{j}^{-}, f_{k}^{+}\right]|0\rangle=p \delta_{j k}|0\rangle, \quad\left(f_{j}^{ \pm}\right)^{\dagger}=f_{j}^{\mp},
$$

and by irreducibility under the action of the Lie algebra generated by the elements $f_{j}^{+}$, $f_{j}^{-}(j=1,2, \ldots)$, subject to $(1)$, i.e. the Lie algebra $\mathfrak{s o}(\infty)$. In $(15), A^{\dagger}$ is the Hermitian adjoint of the operator $A$ with respect to the inner product in the representation space $W(p)$. Following the common terminology, such representations $W(p)$ are called unitary.

Note that for a finite number of parafermion generators $f_{j}^{+}, f_{j}^{-}(j=1,2, \ldots, n)$, the corresponding Fock space $W(p)$ is a finite-dimensional irreducible unitary representation of the Lie algebra $\mathfrak{s o}(2 n+1)$. The structure of $W(p)$, and in particular the parafermion operator actions in an appropriate orthogonal basis of $W(p)$, has been determined in [6].

For the infinite rank case, a basis of $W(p)$ consists of all stable GZ-patterns $\mid m$ ) with $m_{i j} \leq p$ for all $i$ and $j$. This basis is orthogonal: $\left(m^{\prime} \mid m\right)=\delta_{m, m^{\prime}}$. The action of the $\mathfrak{s o}(\infty)$ generators $f_{j}^{ \pm}$on the basis vectors $\mid m$ ) is given by

$$
\left.\left.\left.\left.f_{j}^{+} \mid m\right)=\sum_{m^{\prime}}\left(m^{\prime}\left|f_{j}^{+}\right| m\right) \mid m^{\prime}\right), \quad f_{j}^{-} \mid m\right)=\sum_{m^{\prime}}\left(m^{\prime}\left|f_{j}^{-}\right| m\right) \mid m^{\prime}\right)
$$

where these matrix elements are related by $\left(m\left|f_{j}^{-}\right| m^{\prime}\right)=\left(m^{\prime}\left|f_{j}^{+}\right| m\right)$. For a given $\left.\mid m\right)$, there are only a finite number of non-zero matrix elements, so the sums in (16) are finite. Let us describe the action of $f_{j}^{+}$in (16). The only vectors appearing in the right hand side are (stable) GZ-patterns $\mid m^{\prime}$ ) such that:

- For $n<j$, the rows of $\mid m)$ and $\left.\mid m^{\prime}\right)$ are the same: $\left[m^{\prime}\right]^{n}=[m]^{n}$;

- For $n \geq j$, the rows of $\mid m)$ and $\left.\mid m^{\prime}\right)$ differ by 1 for one entry only. More precisely, $\left[\mathrm{m}^{\prime}\right]^{n}$ is the same sequence as $[m]^{n}$, apart from the fact that one entry (say, at position $\langle n\rangle$ ) has been increased by 1 . So for each $n \geq j$, there is a unique index denoted by $\langle n\rangle$, with $\langle n\rangle \in\{1,2, \ldots, n\}$, such that

$$
m_{\langle n\rangle, n}^{\prime}=m_{\langle n\rangle, n}+1 \text { and } m_{i, n}^{\prime}=m_{i, n} \text { for all } i \neq\langle n\rangle .
$$


The explicit expression of the matrix element $\left(m^{\prime}\left|f_{j}^{+}\right| m\right)$ is deduced from that of the finite rank case. Let us assume that $\mid m$ ) is stable with respect to row $N$. For $j \leq N$, one has

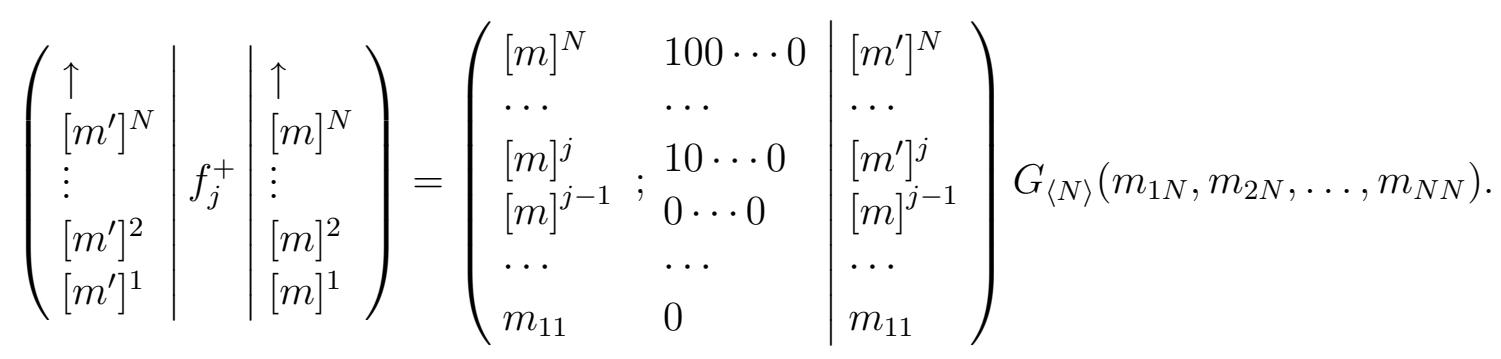

In the right hand side of (18), the first factor is a $\mathfrak{u}(N)$ Clebsch-Gordan coefficient (CGC) [12], of which the expression will be given soon, and $G_{k}\left(m_{1 N}, m_{2 N}, \ldots, m_{N N}\right)$ is given by (see [6, Prop. 4])

$$
\left(-\frac{\left(\mathcal{E}_{N}\left(m_{k N}+N-k\right)+1\right) \prod_{j=1(j \neq k)}^{N}\left(m_{k N}-m_{j N}-k+j\right)}{\prod_{j=1\left(j \neq \frac{k}{2}\right)}^{\lfloor N / 2\rfloor}\left(m_{k N}-m_{2 j, N}-k+2 j\right)\left(m_{k N}-m_{2 j, N}-k+2 j+1\right)}\right)^{1 / 2}
$$

for $k$ even, and by

$$
\left(\frac{\left(p-m_{k N}+k-1\right)\left(\mathcal{O}_{N}\left(m_{k N}+N-k\right)+1\right) \prod_{j=1(j \neq k)}^{N}\left(m_{k N}-m_{j N}-k+j\right)}{\prod_{j=1\left(j \neq \frac{k+1}{2}\right)}^{\Gamma N / 2\rceil}\left(m_{k N}-m_{2 j-1, N}-k+2 j-1\right)\left(m_{k N}-m_{2 j-1, N}-k+2 j\right)}\right)^{1 / 2}
$$

for $k$ odd, where $1 \leq k \leq N$. Herein $\mathcal{E}$ and $\mathcal{O}$ are the even and odd functions defined by $\mathcal{E}_{j}=1$ if $j$ is even and 0 otherwise; $\mathcal{O}_{j}=1$ if $j$ is odd and 0 otherwise. Also, note that a product such as $\prod_{j \neq k=1}^{N}$ means "the product over all $j$-values running from 1 to $N$, but excluding $j=k$ ". The notation $\lfloor a\rfloor$ (resp. $\lceil a\rceil$ ) refers to the floor (resp. ceiling) of $a$, i.e. the largest integer not exceeding $a$ (resp. the smallest integer greater than or equal to $a$ ).

The $\mathfrak{u}(N)$ CGC in (18) (i.e. the first factor of the right hand side of (18)) is given by (see [12] or [7, Appendix A])

$$
\begin{aligned}
& S(\langle N\rangle,\langle N-1\rangle) S(\langle N-1\rangle,\langle N-2\rangle) \ldots S(\langle j+1\rangle,\langle j\rangle)\left(\frac{\prod_{k=1}^{j-1}\left(l_{k, j-1}-l_{\langle j\rangle, j}-1\right)}{\prod_{k=1(k \neq\langle j\rangle)}^{j}\left(l_{k j}-l_{\langle j\rangle, j}\right)}\right)^{1 / 2} \\
& \times \prod_{r=j+1}^{N}\left(\prod_{k=1}^{r-1} \frac{\left(l_{k, r-1}-l_{\langle r\rangle, r}-1\right)}{\left(l_{k, r-1}-l_{\langle r-1\rangle, r-1}-1\right)} \prod_{k=1}^{r} \frac{\left(l_{k r}-l_{\langle r-1\rangle, r-1}\right)}{\left(l_{k r}-l_{\langle r\rangle, r}\right)}\right)^{1 / 2},
\end{aligned}
$$

where, as usual, $l_{i j}=m_{i j}-i$ and $S(k, r)=1$ for $k \leq r$ and $S(k, r)=-1$ for $k>r$.

Secondly, for $j>N$, take $j$ as the stable index for $\mid m$ ) and apply again (18) (clearly, if $(m)$ is stable with respect to $N$, then it is also stable with respect to $j>N$ ).

After this rather technical summary, let us state the main result. 
Theorem 6 The $\mathfrak{s o}(\infty)$ representation $W(p)$, i.e. the parafermion Fock representation of order $p$, has an orthonormal basis consisting of all stable GZ-patterns with entries at most p. The action of the $\mathfrak{s o}(\infty)$ generators $f_{j}^{ \pm}$on the basis vectors $\left.\mid m\right)$ is given by (16), where the actual matrix elements are presented in (18). Under this action, $W(p)$ is a unitary irreducible $\mathfrak{s o}(\infty)$ representation, generated by the vacuum vector $|0\rangle=\mid 0)$ consisting of the GZ-pattern with all zero entries.

For details of the proof, see [13]. Because of the correspondence described in the previous section, this can also be stated as

Corollary 7 The $\mathfrak{s o}(\infty)$ irreducible representation $W(p)$ has an orthonormal basis consisting of all SSYT with entries from $\mathbb{Z}_{+}$and with the width of the Young diagram at most $p$.

The matrix elements in (18) look complicated at first sight, but in fact they are not so difficult to use explicitly. Let us give an example, and compute the action of $f_{1}^{+}, f_{2}^{+}, \ldots$ on the vector $(13)$ of $W(p)$ (where, obviously, $p \geq 5$ ). One finds:

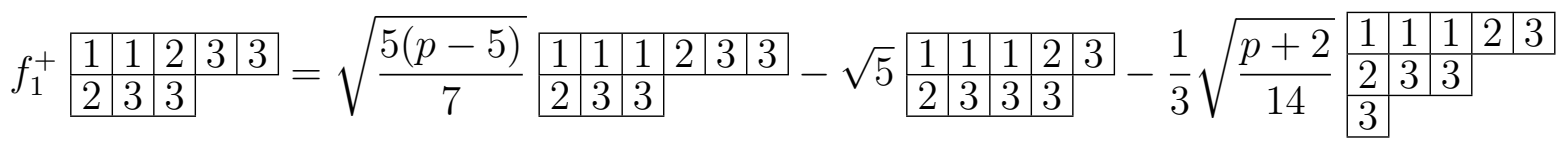

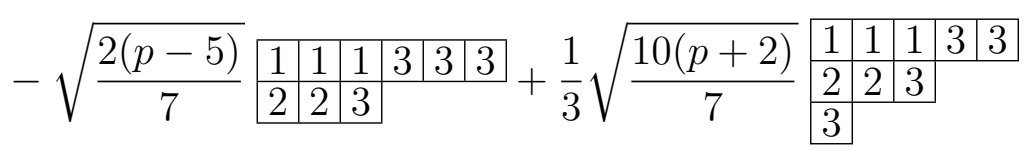

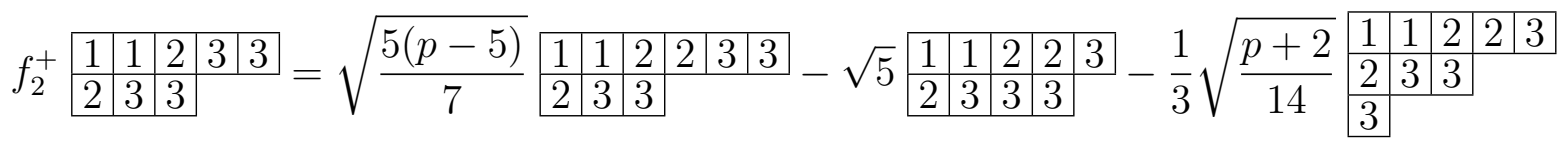

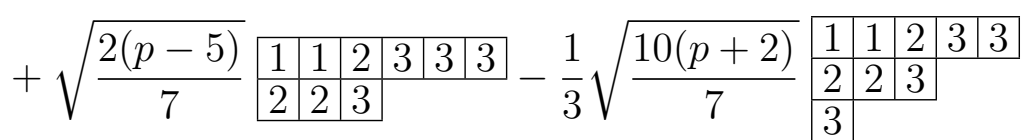

$$
\begin{aligned}
& f_{3}^{+} \begin{array}{|l|l|l|l|l}
1 & 1 & 2 & 3 & 3
\end{array}=\sqrt{\frac{18(p-5)}{7}} \begin{array}{|l|l|l|l|l|l|l|l|l|l|}
1 & 18 & 2 & 3 & 3 & 3 \\
2 & 3 & 3 & 3 & &
\end{array}
\end{aligned}
$$

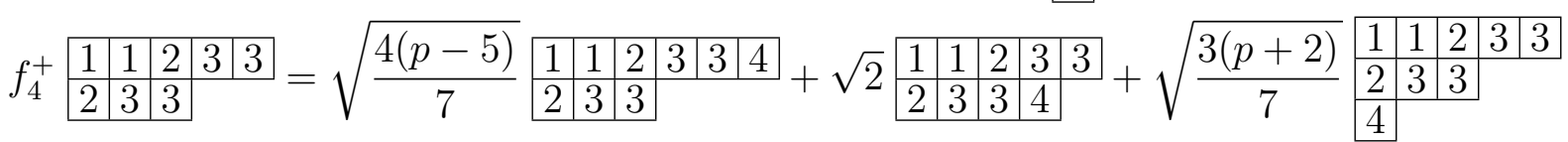

Note that for $p=5$ some matrix elements become automatically zero, corresponding to the coefficients in front of vectors that do not belong to $W(5)$. Also note that, when $f_{j}^{+}$acts on a tableau $T$, the tableaux $T^{\prime}$ appearing in the right hand side satisfy:

- the Young diagram of $T^{\prime}$ consist of that of $T$ with one extra box added in a row;

- the entries of the tableaux $T^{\prime}$ are those of $T$ with an extra entry number $j$, and the positions of the entries $\{1,2, \ldots, j-1\}$ in $T$ are not changed. 
The actual coefficients in front of the tableaux follow from (18). So in this way one has an action of parafermion operators $f_{i}^{ \pm}(i=1,2, \ldots)$ on semi-standard Young tableaux of width at most $p$, which corresponds to an irreducible representation of $\mathfrak{s o}(\infty)$.

Let us also give a simpler example, namely the action of the operators $f_{i}^{ \pm}$on tableaux with only one entry. First of all, note that

$$
\left.f_{i}^{+} \mid 0\right)=\sqrt{p} i
$$

Then, one finds:

$$
\begin{array}{ll}
f_{j}^{+} i=\sqrt{p-1}|j| i-\frac{j}{i} & \text { for } j<i ; \\
f_{j}^{+} i=\sqrt{2(p-1)} i \frac{i}{i} \quad & \text { for } j=i ; \\
f_{j}^{+} i=\sqrt{p-1} i j+\frac{i}{j} & \text { for } j>i .
\end{array}
$$

The case of the paraboson algebra Fock space is similar. We shall describe only partially the results; for more details see [13]. The paraboson Fock space $V(p)$ of order $p$, with $p$ any positive integer, is the Hilbert space with unique vacuum vector $|0\rangle$, defined by means of $(j, k=1,2, \ldots)[2]$

$$
\langle 0 \mid 0\rangle=1, \quad b_{j}^{-}|0\rangle=0, \quad\left\{b_{j}^{-}, b_{k}^{+}\right\}|0\rangle=p \delta_{j k}|0\rangle, \quad\left(b_{j}^{ \pm}\right)^{\dagger}=b_{j}^{\mp},
$$

and by irreducibility under the action of the Lie superalgebra $\mathfrak{o s p}(1 \mid \infty)$. For a finite number of paraboson operators $b_{j}^{+}, b_{j}^{-}(j=1,2, \ldots, n)$, the corresponding Fock space $V(p)$ is an infinite dimensional irreducible unitary representation of the Lie superalgebra $\mathfrak{o s p}(1 \mid 2 n)$, which has been studied in detail in [7]. For the infinite rank case, a basis of $V(p)$ consists of all stable GZ-patterns $\mid m)$ with $m_{i j}=0$ whenever $i>p$. In other words, the GZpatterns have only $p$ columns, the rest of the columns consisting of zeros only. This basis is orthogonal. The action of the $\mathfrak{o s p}(1 \mid \infty)$ generators $b_{j}^{ \pm}$on the basis vectors $\left.\mid m\right)$ is given by

$$
\left.\left.\left.\left.b_{j}^{+} \mid m\right)=\sum_{m^{\prime}}\left(m^{\prime}\left|b_{j}^{+}\right| m\right) \mid m^{\prime}\right), \quad b_{j}^{-} \mid m\right)=\sum_{m^{\prime}}\left(m^{\prime}\left|b_{j}^{-}\right| m\right) \mid m^{\prime}\right),
$$

where these matrix elements are related by $\left(m\left|b_{j}^{-}\right| m^{\prime}\right)=\left(m^{\prime}\left|b_{j}^{+}\right| m\right)$. The sums in (23) are again finite. The action of $b_{j}^{+}$in (23) is easy to describe: the same (stable) GZ-patterns $\mid m^{\prime}$ ) appear in the right hand side of (23) as in the case of parafermions (but $\langle n\rangle \in\{1, \ldots, p\}$ in the description (17)). Only the expression of the matrix element $\left(m^{\prime}\left|b_{j}^{+}\right| m\right)$ itself is different, 
compared to (18). In fact, the expression of $\left(m^{\prime}\left|b_{j}^{+}\right| m\right)$ has the same structure as (18), but with the function $G$ replaced by (without loss of generality we may assume that $N \geq p$ )

$$
\begin{aligned}
\tilde{F}_{k}\left(m_{1 N}, m_{2 N}, \ldots, m_{p N}\right)= & (-1)^{m_{k+1, N}+\cdots+m_{p N}}\left(m_{k N}+p+1-k\right)^{1 / 2} \\
& \times \prod_{j=1}^{p}\left(\frac{m_{j N}-m_{k N}-j+k}{m_{j N}-m_{k N}-j+k-\mathcal{O}_{m_{j N}-m_{k N}}}\right)^{1 / 2} .
\end{aligned}
$$

We can now state:

Theorem 8 The osp $(1 \mid \infty)$ representation $V(p)$, i.e. the paraboson Fock representation of order $p$, has an orthonormal basis consisting of all stable GZ-patterns with at most $p$ nonzero entries per row. The action of the $\mathfrak{o s p}(1 \mid \infty)$ generators $b_{j}^{ \pm}$on the basis vectors $\left.\mid m\right)$ is given by (23), where the actual matrix elements are obtained by means of (24). Under this action, $V(p)$ is a unitary irreducible osp $(1 \mid \infty)$ representation, generated by the vacuum vector $|0\rangle=\mid 0)$ consisting of the GZ-pattern with all zero entries.

Using the correspondence with Young tableaux, one finds:

Corollary 9 The osp $(1 \mid \infty)$ irreducible representation $V(p)$ has an orthonormal basis consisting of all SSYT with entries from $\mathbb{Z}_{+}$and with height of the Young diagram at most p.

Let us again give a simple example:

$$
\left.b_{i}^{+} \mid 0\right)=\sqrt{p} i
$$

and

$$
\begin{array}{ll}
b_{j}^{+} i=\sqrt[j]{j}-\sqrt{p-1} \frac{j}{i} & \text { for } j<i ; \\
b_{j}^{+} i=\sqrt{2} i i & \text { for } j=i ; \\
b_{j}^{+} i=i j+\sqrt{p-1} \frac{i}{j} & \text { for } j>i .
\end{array}
$$

The situation is similar to the action of the parafermion generators. The coefficients in front of the tableaux however, are different. They take into account that the height of the tableaux appearing in the right hand side does not exceed $p$. 


\section{SUMMARY}

In this paper we have extended the results of $[6,7]$ and constructed representations of order $p$ for an infinite set of parafermions and parabosons. The corresponding algebras are the infinite rank Lie algebra $\mathfrak{s o}(\infty)$ and the infinite rank Lie superalgebra $\mathfrak{o s p}(1 \mid \infty)$. The Fock spaces of these algebras are described in terms of stable infinite GZ-patterns or SSYT. These are irreducible $\mathfrak{s o}(\infty)$ or $\mathfrak{o s p}(1 \mid \infty)$ representations, and the actions of the generators on basis elements is given explicitly.

It is worth mentioning that the Fock spaces $W(p)$ and $V(p)$ also have interesting characters, which can be given as series of Schur functions. For more details on this, and a character formula, see [13].

NIS was supported by a project from the Fund for Scientific Research - Flanders (Belgium) and by project P6/02 of the Interuniversity Attraction Poles Programme (Belgian State - Belgian Science Policy).

[1] H.S. Green, Phys. Rev. 90, 270 (1953).

[2] O.W. Greenberg and A.M.L. Messiah, Phys. Rev. B 138, 1155 (1965).

[3] S. Kamefuchi and Y. Takahashi, Nucl. Phys. 36, 177 (1962).

[4] C. Ryan, and E.C.G. Sudarshan, Nucl. Phys. 47, 207 (1963).

[5] A.Ch. Ganchev and T.D. Palev, J. Math. Phys. 21, 797 (1980).

[6] N.I. Stoilova and J. Van der Jeugt, J. Phys A: Math. Theor. 41, 075202 (2008).

[7] S. Lievens, N.I. Stoilova and J. Van der Jeugt, Commun. Math. Phys. 281, 805 (2008).

[8] V.G. Kac, Infinite dimensional Lie algebras (Cambridge University Press, 1990).

[9] V.G. Kac, Adv. Math. 26, 8 (1977).

[10] I.M. Gel'fand and M.L. Zetlin, Dokl. Akad. Nauk SSSR 71, 825 (1950).

[11] I.G. Macdonald, Symmetric Functions and Hall Polynomials (Oxford University Press, Oxford, 2nd edition, 1995).

[12] N.Ja. Vilenkin and A.U. Klimyk, Representation of Lie Groups and Special Functions, Vol. 3 (Kluwer Academic Publishers, Dordrecht, 1992).

[13] N.I. Stoilova and J. Van der Jeugt, Int. J. Math., in press (2009). 